\title{
A refined search for pulsations in white dwarf companions to millisecond pulsars ${ }^{\star}$
}

\author{
Mukremin Kilic,,${ }^{1,2} \dagger$ J. J. Hermes, ${ }^{3} \ddagger$ A. H. Córsico, ${ }^{4}$ Alekzander Kosakowski, ${ }^{1}$ \\ Warren R. Brown, ${ }^{5}$ John Antoniadis, ${ }^{6}$ Leila M. Calcaferro, ${ }^{4}$ A. Gianninas, ${ }^{1}$ \\ Leandro G. Althaus ${ }^{4}$ and M. J. Green ${ }^{7}$ \\ ${ }^{1}$ Department of Physics and Astronomy, University of Oklahoma, 440 W. Brooks St., Norman, OK 73019, USA \\ ${ }^{2}$ Institute for Astronomy, University of Edinburgh, Royal Observatory, Blackford Hill, Edinburgh EH9 $3 H J$, UK \\ ${ }^{3}$ Department of Physics and Astronomy, University of North Carolina, Chapel Hill NC 27599, USA \\ ${ }^{4}$ Facultad de Ciencias Astronómicas y Geofísicas (UNLP), La Plata, Argentina \\ ${ }^{5}$ Smithsonian Astrophysical Observatory, 60 Garden St, Cambridge MA 02138, USA \\ ${ }^{6}$ Dunlap Institute for Astronomy \& Astrophysics, University of Toronto, 50 St. George Street, Toronto M5S 3 H4, Canada \\ ${ }^{7}$ Astronomy and Astrophysics Group, Department of Physics, University of Warwick, Gibbet Hill Road, Coventry CV4 7AL, UK
}

Accepted 2018 June 7. Received 2018 May 27; in original form 2018 April 11

\begin{abstract}
We present optical high-speed photometry of three millisecond pulsars with low-mass $\left(<0.3 \mathrm{M}_{\odot}\right)$ white dwarf companions, bringing the total number of such systems with follow-up time-series photometry to five. We confirm the detection of pulsations in one system, the white dwarf companion to PSR J1738+0333, and show that the pulsation frequencies and amplitudes are variable over many months. A full asteroseismic analysis for this star is underconstrained, but the mode periods we observe are consistent with expectations for an $M_{\star}=0.16-0.19 M_{\odot}$ white dwarf, as suggested from spectroscopy. We also present the empirical boundaries of the instability strip for low-mass white dwarfs based on the full sample of white dwarfs, and discuss the distinction between pulsating low-mass white dwarfs and subdwarf A/F stars.
\end{abstract}

Key words: stars: oscillations - stars: variables: general - white dwarfs - pulsars: individual: PSR J1738+0333, PSR J1911- 5958A, PSR J2234+0611.

\section{INTRODUCTION}

White dwarfs (WDs) are the most common type of companion detected around millisecond pulsars (MSPs, Lorimer 1998), and thus play a crucial role in establishing the mass and equation of state of neutron stars. Pulsating WDs can provide a second clock in these systems, constraining pulsar spin-down and magnetic-field decay (e.g. Kulkarni 1986). The characteristic MSP spin-down ages do not necessarily represent their true ages (Tauris 2012). Hence, cooling ages of their WD companions provide the only reliable age measurements in these systems (Istrate et al. 2014).

\footnotetext{
* Based on observations obtained at the Gemini Observatory, which is operated by the Association of Universities for Research in Astronomy, Inc., under a cooperative agreement with the NSF on behalf of the Gemini partnership: the National Science Foundation (United States), the National Research Council (Canada), CONICYT (Chile), the Australian Research Council (Australia), Ministério da Ciência, Tecnologia e Inovação (Brazil) and Ministerio de Ciencia, Tecnología e Innovación Productiva (Argentina). $\dagger$ E-mail: kilic@ou.edu

$\ddagger$ Hubble Fellow
}

Pulsation frequencies and amplitudes depend on the internal structure of the WD, and thus its evolutionary age (Winget \& Kepler 2008). We present an observational study of MSPs with low-mass WD companions with effective temperatures in the regime where pulsations may be excited by the onset of a surface convection zone. Hydrogen-dominated WDs with canonical carbon-oxygen cores $\left(M_{\star} \sim 0.6 \mathrm{M}_{\odot}\right)$ exhibit gravity-mode pulsations between effective temperatures of $\approx 10,500$ and $13,000 \mathrm{~K}$. Detailed asteroseismological analyses of these stars provide unique constraints on the core carbon-oxygen ratio (Giammichele et al. 2017), as well as the thickness of the surface hydrogen and helium layers, which regulate the cooling of the star.

Following the discovery of several extremely low-mass (ELM, $M_{\star} \lesssim 0.3 M_{\odot}$ ) WDs in the field (Kilic et al. 2010) and as companions to MSPs, Steinfadt, Bildsten \& Arras (2010) predicted that ELM WDs should also pulsate in a similar temperature range (see also Córsico et al. 2012; Van Grootel et al. 2013). Their initial search did not find any pulsators (Steinfadt et al. 2012), but later searches by Hermes et al. (2012, 2013a,b) found oscillations in several ELM WDs with pulsation periods ranging from about $20 \mathrm{~min}$ to more than an hour. 
However, the instability strip for low-mass WDs is complicated by an overlapping population of subdwarf A-type (sdA) stars, stars that have spectroscopic surface gravities comparable to WDs (Kepler et al. 2015, 2016) but which may be mostly metal-poor mainsequence stars (Brown, Kilic \& Gianninas 2017; Pelisoli, Kepler $\&$ Koester 2018). For example, Bell et al. (2017) found a $4.3 \mathrm{~h}$ dominant pulsation mode in J1355+1956, a star which has $T_{\text {eff }}=$ $8050 \pm 120 \mathrm{~K}$ and $\log g=6.10 \pm 0.06$ based on pure hydrogen atmosphere models. However, this period significantly exceeds the theoretical limit for pulsations in ELM WDs (Córsico \& Althaus 2016; Bell et al. 2017). J1355+1956 is better understood as a SX Phe or $\delta$ Scuti variable (Brown et al. 2017).

Given the problems with distinguishing bona-fide pulsating lowmass WDs and pulsating sdA-type stars based on optical spectroscopy, low-mass WD companions to MSPs provide a more reliable opportunity to constrain the boundaries of the ZZ Ceti instability strip for these stars. Because neutron stars are spun up to millisecond periods through accretion in a compact binary system, MSP companions are expected to be low-mass white dwarfs, and not sdA stars.

There are currently five companions to MSPs with spectroscopic temperatures and surface gravities within $1500 \mathrm{~K}$ of the extended, low-mass ZZ Ceti instability strip. Kilic et al. (2015) discovered pulsations in the WD companion to PSR J1738+0333. Here, we present the results from Gemini follow-up photometry of two additional MSP companions, and additional observations of $\mathrm{J} 1738+0333$ from three ground-based facilities. We describe the results for PSR J1911-5958A, PSR J2234+0611, and PSR J1738+0333 in Sections 2-4, respectively. We discuss the constraints on the instability strip for low-mass WDs in Section 5 and conclude.

\section{PSR J1911-5958A}

PSR J1911-5958A is a $3.3 \mathrm{~ms}$ pulsar with a $B=22.2 \mathrm{mag}$ WD companion in a $0.87 \mathrm{~d}$ orbit (D'Amico et al. 2001). Bassa et al. (2006) used optical spectroscopy of the companion to constrain its parameters, $T_{\text {eff }}=10090 \pm 150 \mathrm{~K}$ and $\log g=6.44 \pm 0.20$. Given the recent improvements in the Stark broadening calculations of the hydrogen lines in dense plasmas by Tremblay \& Bergeron (2009), we refit the same spectrum (kindly provided to us by C. Bassa) with 1D pure hydrogen model atmospheres that include these improvements (Gianninas et al. 2014). The best-fit model has $T_{\text {eff }}=10270 \pm 140 \mathrm{~K}$ and $\log g=6.72 \pm 0.05$, and the Tremblay et al. (2015) 3D model corrections change these parameters to $T_{\text {eff }}$ $=9980 \pm 140 \mathrm{~K}$ and $\log g=6.65 \pm 0.05$.

Bassa et al. (2006) used $23 \times 600 \mathrm{~s}$ and $30 \times 30 \mathrm{~s} \mathrm{B-band}$ acquisition images to check for optical variability, but found no significant variations. They found an rms scatter of 0.02-0.05 mag for the WD, but this was comparable to the scatter seen in reference stars of similar brightness. Steinfadt et al. (2012) obtained Hubble Space Telescope observations of PSR J1911-5958A over four orbits, but with gaps in the data due to occultations by Earth. They ruled out pulsations with amplitudes larger than 16 mmag.

We obtained time-series photometry of PSR J1911-5958A using the 8-m Gemini South telescope with the Gemini Multi-Object Spectrograph (GMOS) on UT 2015 June 17 as part of the queue program GS-2015A-Q-81. We obtained $88 \times 105 \mathrm{~s}$ exposures through an SDSS- $g$ filter over $3.0 \mathrm{~h}$. To reduce the read-out time and telescope overhead to $\approx 15$ s, we binned the chip by $4 \times 4$, which resulted in a plate scale of $0.3^{\prime \prime}$ pixel $^{-1}$. Observations were obtained under thin cirrus with a median seeing of $1.0^{\prime \prime}$. We used the

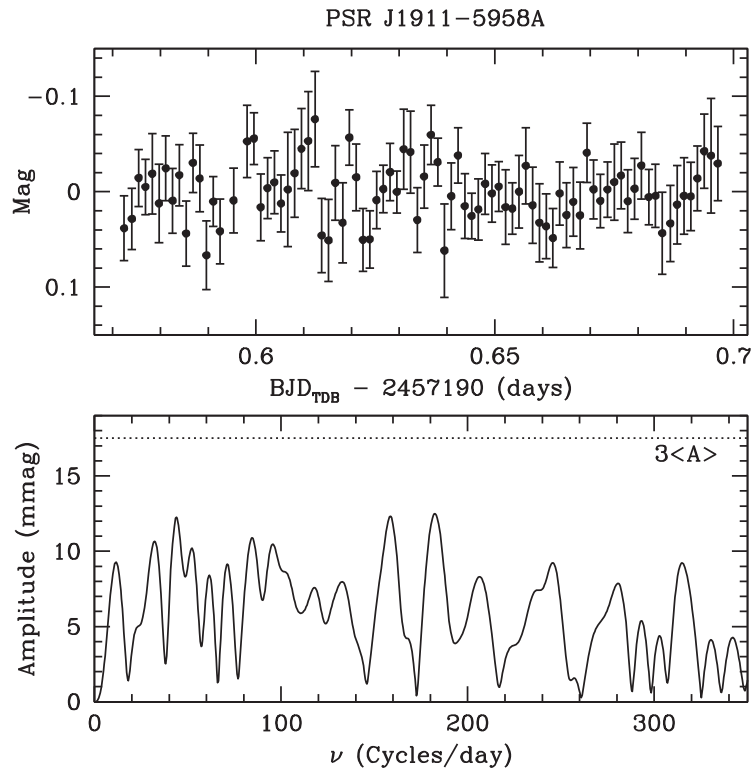

Figure 1. The Gemini South light curve (top panel) and its Fourier transform (bottom panel) for the optical companion to PSR J1911-5958A. The dashed line marks the $3 \mathrm{~A}$ significance level (17.4 mmag), as described in the text.

standard IRAF Gemini GMOS routines and the daily bias and sky flats to reduce and calibrate the data, and corrected our times to the solar-system barycentre using the tools of Eastman, Siverd \& Gaudi (2010). PSR J1911-5958A has a relatively bright source 3" away. To minimize contamination from this nearby source, we performed point spread function photometry, and used 15 non-variable reference stars to calibrate the differential photometry. Given the colour differences between the WD and the relatively red reference stars, we fit a second-degree polynomial to the light curve to remove the long-term trend due to differential extinction.

Fig. 1 shows the Gemini light curve and its Fourier transform for the ELM WD companion to PSR J1911-5958A. The median amplitude A in the Fourier transform is $5.8 \mathrm{mmag}$, but there are no frequency peaks above $12.5 \mathrm{mmag}$. Hence, there is no evidence of pulsations in PSR J1911-5958A. All but one of the known pulsating ELM WDs in short-period binary systems show pulsations with amplitude larger than this limit. The exception is SDSS J1112+1117, which displays pulsations with a maximum amplitude of $8.1 \mathrm{mmag}$ (Hermes et al. 2013a). Such low-level variations would not be detectable for our data on PSR J1911-5958A.

\section{PSR J2234+0611}

PSR J2234+0611 is a 3.6-ms pulsar with a $g=22.2$ mag companion in an eccentric, $e=0.13,32.0 \mathrm{~d}$ orbit (Deneva et al. 2013). Antoniadis et al. (2016) performed synthetic photometry of this target by convolving their $26 \times 1420$ s follow-up spectra with the $g$-band filter response curve. They found peak-to-peak variations of about 0.5 mag in the synthetic photometry, with no obvious correlation with the orbital motion, suggesting that the WD companion in this system may display high-amplitude pulsations.

We obtained time-series photometry of PSR J2234+0611 using the same setup as in Section 2, but on the 8-m Gemini North telescope on UT 2016 July 11 as part of the queue program GN2016B-Q-13. We obtained $148 \times 75 \mathrm{~s} g$-band exposures over $3.7 \mathrm{~h}$. Conditions were photometric with a median seeing of $0.6^{\prime \prime}$. We 

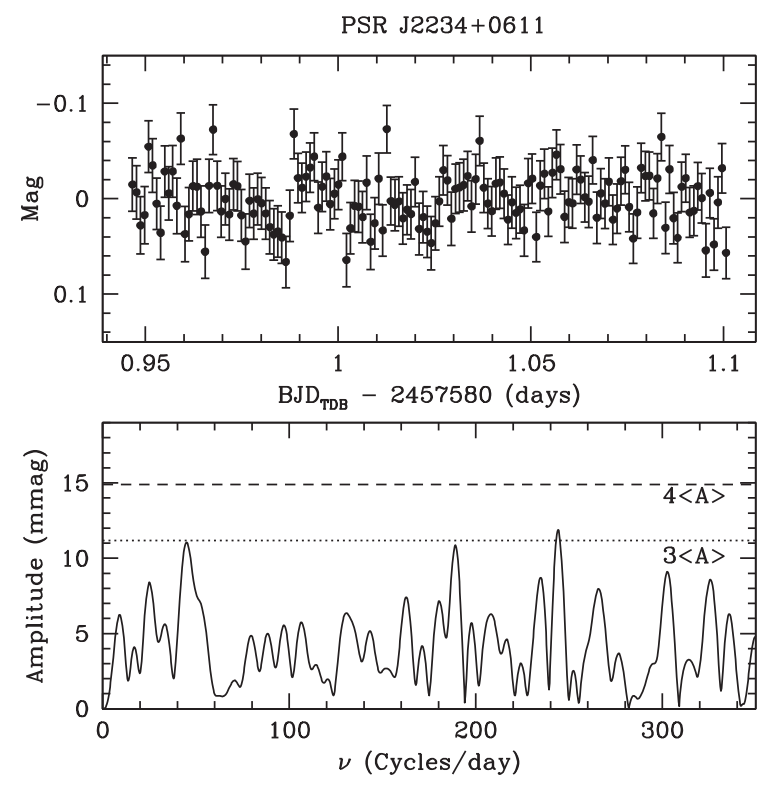

Figure 2. The Gemini North light curve (top panel) and its Fourier transform (bottom panel) for the optical companion to PSR J2234+0611. The dotted and dashed lines mark the $3 \mathrm{~A}$ and $4 \mathrm{~A}$ significance levels, respectively.

performed aperture photometry on PSR J2234+0611 and seven nearby reference stars to calibrate the photometry.

Fig. 2 shows the Gemini light curve and its Fourier transform for the companion to PSR J2234+0611. There are no significant variations down to a $4 \mathrm{~A}$ limit of 14.9 mmag for this WD, ruling out pulsations above this level. Hence, the high-amplitude variations seen in the synthetic photometry of Antoniadis et al. (2016) were likely not intrinsic to the source, and instead likely caused by differential refraction effects and/or variable slit-losses.

\section{PSR J1738+0333}

Unlike PSR J1911-5958A and PSR J2234+0611, the companion to PSR J1738+0333 ( $V=21.3 \mathrm{mag})$ pulsates. Based on $243 \times 50 \mathrm{~s}$ exposures obtained over $5.5 \mathrm{~h}$ in 2014 , Kilic et al. (2015) detected three significant periodicities in the companion to PSR J1738+0333 with 10-15 mmag amplitudes.

To generate a better census of the periods of variability excited in order to complete an asteroseismic analysis and better constrain the interior structure of this pulsating WD, we obtained follow-up observations of PSR J1738+0333 from three different ground-based optical facilities. Unfortunately, weather and poor seeing conspired to challenge two of these data sets.

We were awarded three nights through ESO, from 7 to 9 July 2016, to observe PSR J1738+0333 with ULTRACAM (Dhillon et al. 2007) mounted as a visitor instrument on the 3.5-m New Technology Telescope (NTT) in La Silla, Chile. These data were taken simultaneously through $u^{\prime}, g$ ', $r$ ' filters and reduced using the ULTRACAM pipeline software, with standard bias correction and flat-fielding. We performed variable aperture photometry scaled according to the full width at half maximum and divided our light curve by two brighter nearby comparison stars. However, we obtained less than $1.5 \mathrm{~h}$ of ULTRACAM data in cloudy conditions. We binned our 15-s exposures by 6 , and our light curve obtained through the $g$ ' filter had the highest signal-to-noise; still, we did not see any coherent variability in our ULTRACAM data above a $3 \mathrm{~A}$ limit of $51 \mathrm{mmag}$.

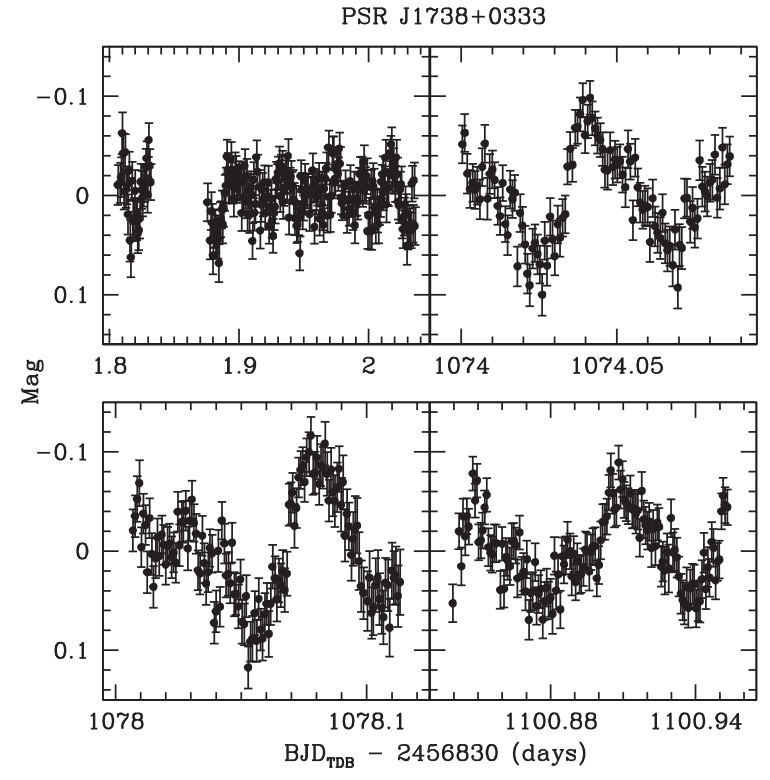

Figure 3. The Gemini North light curve for the optical companion to PSR J1738+0333 from one night in 2014 (top left panel) and three nights in 2017.

We also obtained time-series photometry using the SALTICAM instrument (O'Donoghue et al. 2006) on the 9.2-m South African Large Telescope (SALT) on six visits over five nights: UT 2016 May 3, June 7, June 9, June 29, and July 4. Given the unique tracklength constraints of observing with SALT, our median observing length in a given night was only $33 \mathrm{~min}$. On 2016 June 7, we obtained back-to-back tracks for a total of $89 \mathrm{~min}$ on target. For each visit, we used 45-s exposures obtained through an SDSS- $g$ ' filter. Seeing for each visit ranged from $1.8^{\prime \prime}, 1.6^{\prime \prime}, 1.5^{\prime \prime}, 1.5^{\prime \prime}$, and 1.8", respectively.

We extracted fixed-aperture photometry from the pipelineprocessed SALTICAM data, which is bias- and flat-field corrected. Only the 2016 June 7 data cover a full cycle of the pulsations detected from the 2014 Gemini data set, and so our SALT data are complicated by long-term atmospheric effects. A Fourier transform of the data from 2016 June 7 shows a strong peak at $1213 \pm 28 \mathrm{~s}$ with 23 mmag amplitude, but this peak is not formally significant at 3.4A. As with the ULTRACAM data, our SALTICAM photometry does not well-constrain the long pulsation periods present in PSR J1738+0333.

Our most useful data were obtained using Gemini North on UT 2017 May 30, June 2, June 3, and June 26. We obtained a total of $396 \times 50$ s $g$-band exposures. However, there are only 18 observations from June 2, and given the relatively short baseline of the observations, we exclude those data from our analysis. The other three nights have time baselines of $2.1,2.5$, and $2.7 \mathrm{~h}$, respectively. Only observations from 2017 May 30 were obtained under photometric conditions, and the remaining data were obtained under thin cirrus with a median seeing of $0.6^{\prime \prime}$.

Fig. 3 shows the Gemini light curve of the WD companion to PSR J1738+0333, including the discovery observations from 2014 (top left panel) and the new data from 2017. At least one of the pulsation amplitudes have significantly increased in the 2017 data compared to the previous observations. In fact, PSR J1738+0333 now shows 0.2 mag peak-to-peak variations. Interestingly, Antoniadis et al. (2012) noted $\sim 0.05$ mag scatter in their spectroscopic acquisition images of PSR J1738+0333 from 2006. Hence, the 


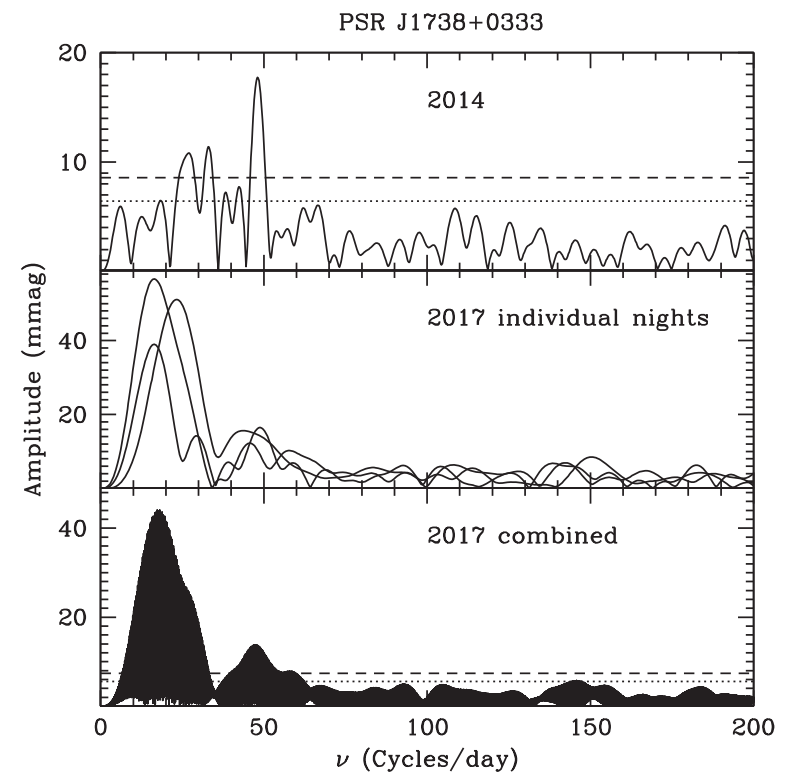

Figure 4. Fourier transforms of the optical counterpart to PSR J1738+0333 show significant frequency and/or amplitude variability from 2014 to 2017. This is not unusual for cooler pulsating WDs of canonical $\left(\sim 0.6 \mathrm{M}_{\odot}\right)$, but is the most extreme example of amplitude variability for a pulsating ELM WD.

Table 1. Multimode frequency solutions for the WD companion to PSR J1738+0333.

\begin{tabular}{lccccc}
\hline Dataset & $\begin{array}{c}\text { Frequency } \\
\text { (Cycles/day) }\end{array}$ & $\begin{array}{c}\text { Amplitude } \\
(\mathrm{mmag})\end{array}$ & $\begin{array}{c}\text { Period } \\
(\mathrm{s})\end{array}$ & $\begin{array}{c}\text { Model } \\
(\mathrm{s})\end{array}$ & $\ell$ \\
\hline 2014 & $27.3919_{-0.420}^{+0.3953}$ & $11.1_{-1.3}^{+2.2}$ & 3154.2 & 3151.8 & 1 \\
$\ldots$ & $32.8090_{-0.4881}^{+0.5962}$ & $10.2_{-1.4}^{+2.1}$ & 2633.4 & 2632.9 & 1 \\
$\ldots$ & $48.3072_{-0.2904}^{+0.3338}$ & $15.3_{-1.3}^{+2.0}$ & 1788.6 & 1790.5 & 1 \\
2017 & $8.7598_{-0.0399}^{+0.0422}$ & $16.2_{-2.8}^{+1.6}$ & 9863.2 & $\ldots$ & $\ldots$ \\
$\ldots$ & $17.3475_{-0.0007}^{+0.0008}$ & $43.5_{-3.1}^{+1.4}$ & 4980.6 & 4980.2 & 2 \\
$\ldots$ & $26.0105_{-0.0405}^{+0.0401}$ & $21.6_{-4.5}^{+1.2}$ & 3321.7 & 3323.0 & 1 \\
$\ldots$ & $47.1124_{-0.0017}^{+0.0021}$ & $12.2_{-1.8}^{+1.6}$ & 1833.9 & 1834.6 & 2 \\
\hline
\end{tabular}

pulsation amplitudes are clearly variable over year and decade time-scales.

The coolest carbon-oxygen core pulsating WDs with masses near $0.6 \mathrm{M}_{\odot}$ show amplitude variability; they typically have longer period pulsations (e.g. Kleinman et al. 1998; Hermes et al. 2014). Long-baseline Kepler and $K 2$ observations have shown that the longest period modes appear to lose phase coherence (Hermes et al. 2017), and a similar phenomenon may be going on at the red edge of the ELM WD instability strip.

Fig. 4 shows the Fourier transforms for the 2014 data (top panel), the individual nights from the 2017 Gemini dataset (middle panel), and the combined 2017 dataset along with the 3A and 4A detection limits (bottom panel). Since each night's data only covers about two cycles of pulsations for the dominant mode, the peak of the Fourier transform is not well defined for each night. Combining the data from all three nights of the 2017 data set, we identify four significant periodicities above the 4A limit. However, one of these significant frequencies, 8.76 cycles/day, is identical to our observing window on the night of UT 2017 June 26. Hence, we ignore it in our analysis. We perform 1000 Monte-Carlo simulations to estimate the uncertainties in frequency and amplitude of each mode. Table 1 presents the results from this analysis for both the 2014 and 2017 data sets.

The WD companion to PSR J1738+0333 showed 10-15 mmag pulsations with periods ranging from $1789 \mathrm{~s}$ to $3154 \mathrm{~s}$ in 2014 , and 12-44 mmag pulsations with periods ranging from $1834 \mathrm{~s}$ to $4981 \mathrm{~s}$ in 2017. The pulsation modes at 27.3919 and 26.0105 cycles per day are consistent in frequency within $3 \sigma$. However, the other modes seem to be unstable in frequency and/or amplitude.

We attempted asteroseismology of this star by fitting the observed periodicities with a new set of low-mass WD models that include a range of hydrogen envelope thicknesses. Calcaferro, Córsico \& Althaus (2018) present the details of these models for $0.15-0.44 M_{\odot}$ WDs with hydrogen envelope masses of $10^{-5.8}$ to $10^{-1.7} M_{\star}$. We use the merit function $\chi^{2}$ as defined by equation (2) of Calcaferro, Córsico \& Althaus (2017) to find the best-fit model. Given the unstable periods and amplitudes, we treat the 2014 and 2017 data sets separately.

Table 1 presents the best-fit model periods (the last column) for each data set assuming that all of the observed periods correspond to $g$ modes with $\ell=1$ or 2 . The 2014 data set is best-explained by a model that has $M_{\star}=0.192 M_{\odot}, M_{\mathrm{H}}=9.5 \times 10^{-4} M_{\star}, T_{\text {eff }}=$ $9273 \mathrm{~K}, \log g=6.63$, and three $\ell=1$ pulsation modes at 1790.5 , 2632.9 , and $3151.8 \mathrm{~s}$. The average difference between the observed and predicted periods in this model is $\Delta P=1.6 \mathrm{~s}$. On the other hand, the 2017 dataset is best explained by a model that has $M_{\star}=$ $0.161 M_{\odot}, M_{\mathrm{H}}=1.7 \times 10^{-2} M_{\star}, T_{\text {eff }}=8883 \mathrm{~K}$, and $\log g=6.05$, with a mixture of $\ell=1$ and 2 modes and an average difference in period of $\Delta P=0.8 \mathrm{~s}$. This model implies a WD cooling age of 3 Gyr.

PSR J1738+0333's WD companion has 3D-corrected spectroscopic values of $T_{\text {eff }}=8910 \pm 150 \mathrm{~K}$ and $\log g=6.30 \pm 0.10$ (Tremblay et al. 2015), and an independent estimate of $\log g=$ $6.45 \pm 0.07$ based on the orbital period decay, mass ratio, parallax, and absolute photometry of the system (Antoniadis et al. 2012). Even though the asteroseismological fits confirm the nature of the companion to PSR J1738+0333 as a low-mass WD, the bestfit model depends heavily on the exact values of the periods. For example, the 3321.7 s mode in the 2017 dataset is uncertain by about $10 \mathrm{~s}$. Repeating our asteroseismological analysis of this data set by taking into account the period uncertainties, we find best-fit solutions with $M_{\star}=0.155-0.192 M_{\odot}, M_{\mathrm{H}}=4 \times 10^{-6} M_{\star}$ to $4 \times 10^{-3} M_{\star}$, and cooling ages ranging from 0.03 to $1.4 \mathrm{Gyr}$. This exercise shows that our asteroseismic constraints are not robust, because there are only three pulsation modes detected in each data set with relatively large errors. Combining the results from both the 2014 and 2017 data, we conclude that PSR J1738+0333's companion has $M_{\star}=0.155-0.192 M_{\odot}, T_{\text {eff }}=8840-9270 \mathrm{~K}$, a poorly constrained hydrogen layer mass of $M_{\mathrm{H}} / M_{\star}=4 \times 10^{-6}$ to $1.7 \times 10^{-2}$, and a WD cooling age of 0.03-3 Gyrs.

Gravity-mode pulsations can be driven by tidal excitations in compact binary white dwarf systems (Fuller \& Lai 2011). Tidallyforced oscillations are typically seen in eccentric binaries and with flux variations at integer multiples of the orbital frequency (Fuller \& Lai 2012), which is 2.82 cycles per day for PSR $\mathrm{J} 1738+0333$. Due to the relatively large errors, any of the observed frequencies in the 2014 data set can be explained by tidal excitation. However, the eccentricity of the PSR J1738+0333 binary is very low, $3.5 \pm 1.1 \times 10^{-7}$ (Antoniadis et al. 2012), and none of the pulsation frequencies in the 2017 dataset are multiples of the orbital frequency. Hence, tidal excitation is unlikely to explain the pulsations seen in PSR J1738+0333's WD companion. 
Table 2. 3D-model-corrected parameters of the WD companions to five MSPs.

\begin{tabular}{llcc}
\hline $\begin{array}{l}\text { Object } \\
\text { PSR }\end{array}$ & $\begin{array}{l}T_{\text {eff }} \\
(\mathrm{K})\end{array}$ & $\begin{array}{c}\log g \\
\left(\mathrm{~cm} \mathrm{~s}^{-2}\right)\end{array}$ & Source \\
\hline J1012+5307 & $8500 \pm 120$ & $6.31 \pm 0.09$ & This paper \\
J1738+0333 & $8910 \pm 150$ & $6.30 \pm 0.10$ & Antoniadis et al. (2012) \\
J1909-3744 & $8920 \pm 150$ & $6.81 \pm 0.15$ & Antoniadis (2013) \\
J1911-5958A & $9980 \pm 140$ & $6.65 \pm 0.05$ & This paper \\
J2234+0611 & $8600 \pm 190$ & $6.97 \pm 0.22$ & Antoniadis et al. (2016) \\
\hline
\end{tabular}

\section{THE ZZ CETI INSTABILITY STRIP FOR LOW-MASS WDS}

There are five MSP + ELM WD system where optical spectroscopy puts the WD within $1500 \mathrm{~K}$ of the instability strip for low-mass WDs. We now have high-cadence photometry for all five systems, and only PSR J1738+0333's companion pulsates to detectable amplitudes. Table 2 presents the physical parameters for these five systems based on the 1D model atmosphere fits and the 3D corrections of Tremblay et al. (2015).

The companion to PSR J1012+5307 has two different $\log g$ estimates in the literature, $6.75 \pm 0.07$ (van Kerkwijk, Bergeron \& Kulkarni 1996) and $6.34 \pm 0.20$ (Callanan, Garnavich \& Koester 1998), and both are based on an analysis that does not include the recent Stark broadening profiles of Tremblay \& Bergeron (2009). To improve the constraints on its physical parameters, we obtained four back-to-back 12-15min spectra of PSR J1012+5307 using the MMT Blue Channel Spectrograph equipped with the 832 lines $\mathrm{mm}^{-1}$ grating and the $1^{\prime \prime}$ slit on UT $2016 \mathrm{Dec} 2$. This setup provided spectra with $1 \AA$ resolution over 3600-4500 A. After shifting each spectrum to rest velocity, we fitted the summed spectrum using 1D pure hydrogen model atmospheres that include the improved Stark broadening profiles (Gianninas et al. 2014). The best-fit model has $T_{\text {eff }}=8630 \pm 120 \mathrm{~K}$ and $\log g=6.63 \pm 0.09$, and the 3D model corrections lower these values to $T_{\text {eff }}=8500 \mathrm{~K}$ and $\log g=6.31$, respectively.

Looking at Table 2, the only pulsating companion to a MSP $(\mathrm{J} 1738+0333)$ has $T_{\text {eff }}=8910 \mathrm{~K}$ and $\log g=6.3$. The companion to PSR J1909 - 3744 has an identical temperature to J1738+0333, but its surface gravity is 0.5 dex higher. Similarly, PSR J1012+5307's companion has an identical surface gravity to J1738+0333, but its temperature is about $400 \mathrm{~K}$ cooler. MSP companions cooler than or more massive than $\mathrm{J} 1738+0333$ do not pulsate, and we suspect this pulsating WD is near the cool (red) edge of the extended ZZ Ceti instability strip. As WDs move through the instability strip, their convection zones deepen, driving longer-period pulsations. These long-period pulsations also tend to be the least stable in frequency and amplitude.

Fig. 5 shows the physical parameters of these five MSP + WD systems (filled squares) plus the field ELM WDs with follow-up time-series photometry and spectroscopy. There are four pulsating ELM WDs in short-period binaries and those are marked with blue symbols. These are J1112+1117, J1518+0658, J1840+6423, and PSR J1738+0333's WD companion. The first three have $>5 \sigma$ significant parallaxes (1.2-2.8 mas) in Gaia Data Release 2 (Gaia Collaboration et al. 2018), which confirm them as WDs with absolute Gaia G-band magnitudes of $M_{\mathrm{G}}=8.4-9.8$. The latter has parallax measurements in the radio (Antoniadis et al. 2012). These four pulsators occupy a similar parameter space and there is no question about their nature; they are clearly WDs.

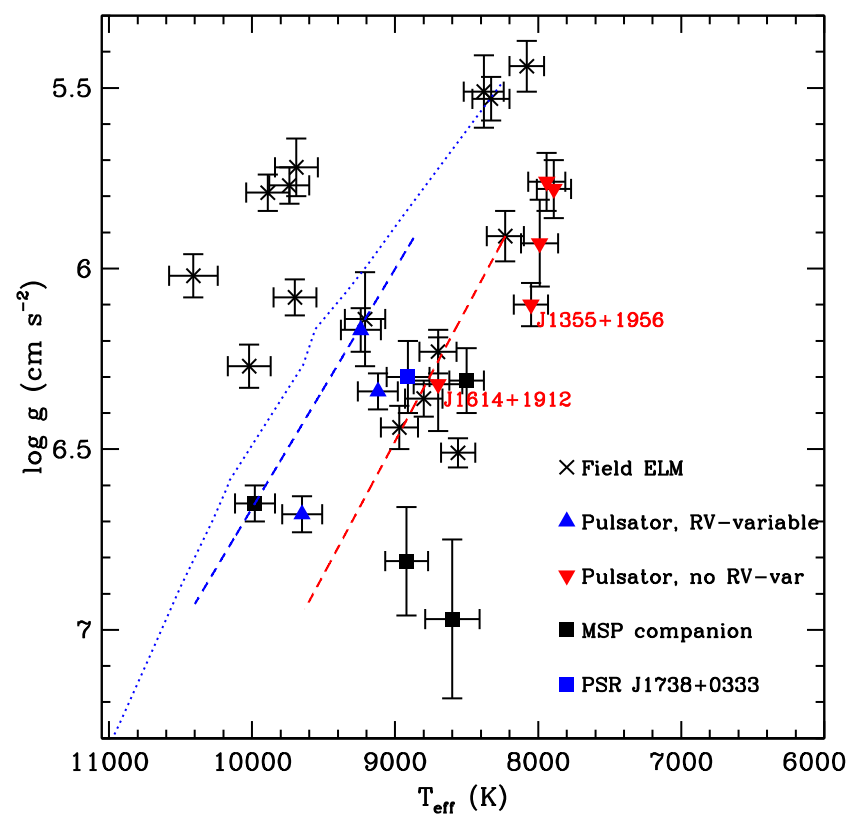

Figure 5. Temperatures and surface gravities (3D corrected) for low-mass WDs with follow-up time-series photometry. Filled squares represent companions to MSPs. Blue symbols mark the pulsating stars that show highamplitude radial velocity (RV) variations since they are in short-period binary systems, while red symbols mark the pulsating stars that are not $\mathrm{RV}$-variable. The dotted line shows the theoretical blue edge of the instability strip from Córsico \& Althaus (2016), and the dashed lines mark our empirical boundaries.

Red symbols show the other five pulsating ELM candidates that are not in short-period binary systems. Brown et al. (2017) demonstrate that unlike the published ELM WD binaries, the majority of the sdA-like objects show no evidence for short-period or highamplitude radial velocity variability. Interestingly, four of these known pulsators are significantly cooler than J1738+0333 and they have $T_{\text {eff }} \approx 8000 \mathrm{~K}$. In addition, Bell et al. (2017) found a dominant 4.3-h pulsation period in one of these stars, J1355+1956, which likely rules out pulsations from a WD and instead favors a high-amplitude $\delta$ Scuti star. The remaining four stars, J1614+1912, $\mathrm{J} 1735+2134, \mathrm{~J} 2139+2227$, and $\mathrm{J} 2228+3623$ also have Gaia parallax measurements (0.18-0.26 mas), which correspond to $M_{\mathrm{G}}=2.4-3.9$ mag. These four objects are too bright to be WDs.

Ignoring these objects, the dashed lines show our empirical boundaries of the instability strip based on the field and MSP + ELM WD samples. The boundaries are

$(\log g)_{\text {blue }}=6.6234 \times 10^{-4}\left(T_{\text {eff }}\right)+0.03987$
$(\log g)_{\text {red }}=7.3754 \times 10^{-4}\left(T_{\text {eff }}\right)-0.15987$.

Given the relatively small number of objects in this figure, these boundaries are preliminary. Nevertheless, our empirical blue edge is consistent with the theoretical predictions from Córsico \& Althaus (2016, shown as a dotted line in Fig. 5).

\section{CONCLUSIONS}

We present high-speed photometry of three MSP + ELM WD systems and find pulsations only in the companion to PSR J1738+0333. We find that the observed modes have variable periods and amplitudes on a time-scale of weeks to years. Our asteroseismic analysis constrains the stellar mass to $0.16-0.19 M_{\odot}$ but provides limited 
constraints on the surface hydrogen layer mass due to the small number of modes observed and the relatively large errors in the measured periods and the paucity of modes observed.

We compare the physical parameters of the MSP + WD systems with the pulsating field ELM WDs. We find two sets of objects, bona-fide pulsating WDs with temperatures near 9,000K and a second set of pulsating stars with temperatures near $8,000 \mathrm{~K}$ that are likely sdA stars. We use the current sample of pulsating and nonpulsating ELM WDs to constrain the boundaries of the instability strip. Our empirical blue edge is consistent with theoretical predictions, but high-speed photometry of additional low-mass WDs with $T_{\text {eff }}=9000-10,000 \mathrm{~K}$ would be helpful for improving these boundaries.

\section{ACKNOWLEDGEMENTS}

We gratefully acknowledge support from the NASA grant NNX14AF65G. Support for this work was provided by NASA through Hubble Fellowship grant \#HST-HF2-51357.001-A, awarded by the Space Telescope Science Institute, which is operated by the Association of Universities for Research in Astronomy, Incorporated, under NASA contract NAS5-26555. MJG acknowledges funding from an STFC studentship via grant ST/N504506/1. MK thanks the University of Edinburgh's Institute for Astronomy Wide Field Astronomy Unit staff for their hospitality during his sabbatical visit.

Some of the observations reported in this paper were obtained with the Southern African Large Telescope under programme 20161-SCI-017, as well as observations made with ESO Telescopes at the La Silla Observatory under programme ID 097.D-0342.

\section{REFERENCES}

Antoniadis J. I., 2013, Ph.D. Thesis, University of Bonn

Antoniadis J., et al., 2012, MNRAS, 423, 3316

Antoniadis J., et al., 2016, ApJ, 830, 36

Bassa C. G., van Kerkwijk M. H., Koester D., Verbunt F., 2006, A\&A, 456, 295

Bell K. J., et al., 2017, ApJ, 835, 180

Brown W. R., Kilic M., Gianninas A., 2017, ApJ, 839, 23

Calcaferro L. M., Córsico A. H., Althaus L. G., 2017, A\&A, 607, A33
Calcaferro L. M., Córsico A. H., Althaus L. G., 2018, A\&A, submitted Callanan P. J., Garnavich P. M., Koester D., 1998, MNRAS, 298, 207

Córsico A. H., Althaus L. G., 2016, A\&A, 585, A1

Córsico A. H., Romero A. D., Althaus L. G., Hermes J. J., 2012, A\&A, 547, A96

D’Amico N., Lyne A. G., Manchester R. N., Possenti A., Camilo F., 2001, ApJ, 548, L171

Deneva J. S., et al., 2013, ApJ, 775, 51

Dhillon V. S., et al., 2007, MNRAS, 378, 825

Eastman J., Siverd R., Gaudi B. S., 2010, PASP, 122, 935

Fuller J., Lai D., 2011, MNRAS, 412, 1331

Fuller J., Lai D., 2012, MNRAS, 420, 3126

Gaia Collaboration et al., 2018, A\&A, in press, preprint (arXiv:1804.09365)

Giammichele N., Charpinet S., Brassard P., Fontaine G., 2017, A\&A, 598, A109

Gianninas A., et al., 2014, ApJ, 794, 35

Hermes J. J., et al., 2012, ApJ, 750, L28

Hermes J. J., et al., 2013, ApJ, 765, 102

Hermes J. J., et al., 2013, MNRAS, 436, 3573

Hermes J. J., et al., 2014, ApJ, 789, 85

Hermes J. J., et al., 2017, ApJS, 232, 23

Istrate A. G., Tauris T. M., Langer N., Antoniadis J., 2014, A\&A, 571, L3

Kepler S. O., et al., 2015, MNRAS, 446, 4078

Kepler S. O., et al., 2016, MNRAS, 455, 3413

Kilic M., Brown W. R., Allende Prieto C., Kenyon S. J., Panei J. A., 2010, ApJ, 716, 122

Kilic M., Hermes J. J., Gianninas A., Brown W. R., 2015, MNRAS, 446, L26

Kleinman S. J., et al., 1998, ApJ, 495, 424

Kulkarni S. R., 1986, ApJ, 306, L85

Lorimer D. R., 1998, Living Reviews in Relativity, 1, 10

O’Donoghue D., et al., 2006, MNRAS, 372, 151

Pelisoli I., Kepler S. O., Koester D., 2018, MNRAS, 475, 2480

Steinfadt J. D. R., Bildsten L., Arras P., 2010, ApJ, 718, 441

Steinfadt J. D. R., et al., 2012, PASP, 124, 1

Tauris T. M., 2012, Science, 335, 561

Tremblay P.-E., Bergeron P., 2009, ApJ, 696, 1755

Tremblay P.-E., et al., 2015, ApJ, 809, 148

Van Grootel V., Fontaine G., Brassard P., Dupret M.-A., 2013, ApJ, 762, 57 van Kerkwijk M. H., Bergeron P., Kulkarni S. R., 1996, ApJ, 467, L89

Winget D. E., Kepler S. O., 2008, ARAA, 46, 157

This paper has been typeset from a $\mathrm{T}_{\mathrm{E}} \mathrm{X} / \mathrm{L} \mathrm{T} \mathrm{E} \mathrm{X}$ file prepared by the author. 